\title{
AN APPROXIMATION THEOREM OF RUNGE TYPE FOR THE HEAT EQUATION
}

\author{
B. FRANK JONES, JR.1
}

ABSTRACT. If $\Omega$ is an open subset of $\mathrm{R}^{n+1}$, the approximation problem is to decide whether every solution of the heat equation on $\Omega$ can be approximated by solutions defined on all of $R^{n+1}$. The necessary and sufficient condition on $\Omega$ which insures this type of approximation is that every section of $\Omega$ taken by hyperplanes orthogonal to the $t$-axis be an open set without "holes," i.e., whose complement has no compact component. Part of the proof involves the Tychon off counterexample for the initial value problem.

1. Introduction. A classical theorem of Runge asserts that if $\Omega$ is an open subset of the complex plane, then a necessary and sufficient condition for $\Omega$ to have the property that for any holomorphic function $f(z)$ on $\Omega$ there exists a sequence of polynomials $P_{j}(z)$ which converges to $f(z)$ locally uniformly on $\Omega$ is that $\Omega$ be simply connected. Or equivalently, we could require that the complement of $\Omega$ have no compact component. See [5, pp. 255264], or [3, pp. 6-9].

Malgrange [4] extended this theorem to a large class of elliptic operators. A particular case of this states that if $\Omega$ is an open subset of $\mathbf{R}^{n}$, then a necessary and sufficient condition for $\Omega$ to have the property that for any harmonic function $u$ on $\Omega$ there exists a sequence of harmonic functions $u_{j}$ on $\mathbf{R}^{n}$ which converges to $u$ locally uniformly on $\Omega$ is that the complement of $\Omega$ have no compact component.

The purpose of this paper is to present the corresponding theorem for the heat operator on $\mathbf{R}^{n+1}$,

$$
H=\Delta-\frac{\partial}{\partial t}=\sum_{i=1}^{n} \frac{\partial^{2}}{\partial x_{i}^{2}}-\frac{\partial}{\partial t} .
$$

In doing this we shall require the counterexample of Tychonoff to uniqueness for the initial value problem for $H$. For $n=1$ the example has the form

$$
u(x, t)=\sum_{k=0}^{\infty} f^{(k)}(t) \frac{x^{2 k}}{(2 k) !},
$$

where $f$ is an appropriate infinitely differentiable function vanishing for $t \leq$ 0 but not identically zero. E.g.,

Received by the editors August 6, 1974 .

AMS (MOS) subject classifications (1970). Primary 35K05, 35A35, 35E 10.

Key words and phrases. Nonuniqueness for initial value problem, simply connected, exponential-polynomial solutions.

1 The research in this paper was supported by National Science Foundation Grant GP.33749. 


$$
f(t)= \begin{cases}e^{-t^{-2}}, & t>0 \\ 0, & t \leq 0 .\end{cases}
$$

See [1, pp. 50-51]. For our purposes it is necessary to require also that $f$ vanish for $t \geq 1$, which we can do. And then for arbitrary $n$ the required function can be obtained in the form $u\left(x_{1}, t\right)$. By a translation in $t$ and rescaling we state this as a

Lemma. For any $\delta>0$ there exists a function $u$ on $\mathbf{R}^{n+1}$ satisfying

(i) $\mathrm{Hu}=0$ on $\mathbf{R}^{n+1}$,

(ii) $u(x, t)=0$ for $|t| \geq \delta$,

(iii) $u(0,0) \neq 0$.

2. Topological preliminaries. We shall be working with an open set $\Omega \subset$ $\mathbf{R}^{n+1}$. For each $t \in \mathbf{R}$ let $\Omega(t)$ be the corresponding section of $\Omega: \Omega(t)=$ $\left\{x \in \mathbf{R}^{n}:(x, t) \in \Omega\right\}$. Thus, $\Omega(t)$ is an open subset of $\mathbf{R}^{n}$. If $K$ is a compact set in $\mathbf{R}^{n+1}$, then the section $K(t)$ is a compact subset of $\mathbf{R}^{n}$.

Definition. Let $C \subset D$, where $C$ is a compact set in $\mathbf{R}^{n}$ and $D$ is an open set in $\mathbf{R}^{n}$. Then we denote by $\widehat{C_{D}}$ the union of $C$ and all components of $D-C$ which are relatively compact in $D$.

This hull $\widehat{C_{D}}$ is of prime importance in the Runge theorem for harmonic functions in $\mathbf{R}^{n}$. It is always a compact set. In case $D$ has the further property that $\mathbf{R}^{n}-\dot{D}$ has no compact component, then $\widehat{C_{D}}=\widehat{C_{\mathbf{R}^{n}}}$. In this case we drop the subscript and simply write $\hat{C}$ for the union of $C$ and all bounded components of $\mathbf{R}^{n}-C$.

Definition. If $K$ is a compact set in $\mathbf{R}^{n+1}$, let

$$
\hat{K}=\{(x, t): x \in \widehat{K(t)}\} \text {. }
$$

Lemma. $K$ is compact.

Proof. Each section $\widehat{K(t)}$ is contained in the convex hull of the section $K(t)$. Also, $K(t)=\varnothing$ for sufficiently large $|t|$. Therefore, $\hat{K}$ is bounded. To prove that $\hat{K}$ is closed, suppose that $(x, t) \notin \hat{K}$. This means $x \notin \widehat{K(t)}$, and this implies that there exists a continuous curve $\gamma$ in $\mathbf{R}^{n}$ which starts at $x$ and tends to $\infty$, lying in the open set $\mathbf{R}^{n}-K(t)$. Choose a closed ball $B C$ $\mathbf{R}^{n}-K(t)$ centered at $x$. Then there exists $\epsilon>0$ such that for $\left|t-t^{\prime}\right|<\epsilon$ the set $K\left(t^{\prime}\right)$ is disjoint from $B$ and the image of $\gamma$. But then for $x^{\prime} \in B$ the point $x^{\prime} \notin K \widehat{\left(t^{\prime}\right)}$. I.e., $B \times(t-\epsilon, t+\epsilon)$ is disjoint from $\hat{K}$. This proves $\mathbf{R}^{n+1}$ $-\hat{K}$ is open. Q.E.D.

\section{The approximation theorem.}

Theorem. Let $\Omega \subset \mathbf{R}^{n+1}$ be open. A necessary and sufficient condition that for any solution $u$ of $H u=0$ on $\Omega$ there exists a sequence $u_{j}$ of solutions of $H u_{j}=0$ on $\mathbf{R}^{n+1}$ which converges to $u$ locally uniformly on $\Omega$ is 
that for every $t \in \mathbf{R}$, the complement of the section $\Omega(t)$ have no compact component.

Proof. Sufficiency. We assume that each $\Omega(t)$ satisfies the condition. By Corollary 3.4.1 of [2] it suffices to consider any compactly supported distribution $\mu$ on $\mathrm{R}^{n+1}$ for which the support of $H^{t} \mu$ is contained in $\Omega$, and to prove that the support of $\mu$ is also contained in $\Omega$. Here $H^{t}$ is the adjoint operator $\Delta+\partial / \partial t$. Let $K$ be the support of $H^{t} \mu$. Since each $\Omega(t)$ has a complement free of compact components, $\widehat{K(t)} \subset \Omega(t)$. Thus, $\hat{K} \subset \Omega$ and $\hat{K}$ is compact. Now $H^{t} \mu=0$ on $\mathbf{R}^{n+1}-K$. Since $H^{t}$ is hypoelliptic, $\mu$ is an infinitely differentiable function on $\mathrm{R}^{n+1}-K$. Then for each $t$ the function $\mu(x, t)$ is an analytic function of $x$ for $x \in \mathbf{R}^{n}-K(t)$ (for solutions of the heat equation are analytic in the space variables) which vanishes for sufficiently large $x$ (because $\mu$ has compact support). But unique continuation then requires $\mu(x, t)=0$ on the domain $\mathbf{R}^{n}-\widehat{K(t)}$. Thus, $\mu=0$ on $\mathbf{R}^{n+1}-\hat{K}$. Thus, the support of $\mu$ is contained in the cormpact set $\hat{K}$.

Necessity. We assume that $\Omega$ does not satisfy the required condition. Therefore, there exists some $t$ such that $\mathbf{R}^{n}-\Omega(t)$ has a compact component $A$. By translation we can assume $0 \in A$ and $t=0$, and we can write $\mathbf{R}^{n}$ $\Omega(0)=A \cup B$, where $A$ and $B$ are disjoint and $B$ is closed. Choose an open set $G$ with compact closure $\bar{G}$ such that $A \subset G \subset \bar{G} \subset \mathbf{R}^{n}-B$. Then the boundary of $G$ is contained in $\Omega(0)$. Thus, $\bar{G} \subset \Omega(0) \cup G$. Thus, there exists an infinitely differentiable $\phi$ on $\mathbf{R}^{n}$ having support $C$ contained in $\Omega(0) \cup$ $G$, and satisfying $\phi \equiv 1$ on $G$. But then the support of the gradient $\nabla \phi$ is contained in $C-G \subset \Omega(0)$. Now there exists $\delta>0$ such that supp $\nabla \phi \times$ $[-\delta, \delta] \subset \Omega$. By the lemma of $\S 1$ there exists a function $u$ on $\mathbf{R}^{n+1}$ such that $H^{t} u \equiv 0, u(x, t)=0$ for $|t| \geq \delta, u(0,0)=1$. Let $v(x, t)=\phi(x) u(x, t)$. Then

$$
\left.H^{t} v=2 \nabla \phi \cdot \nabla u+u \cdot\right\lrcorner \phi
$$

so that the support of $H^{t} v$ is contained in supp $\nabla \phi \times[-\delta, \delta]$, which is contained in $\Omega, v$ itself has compact support and $v(0,0)=1$.

But then $H^{t} v$ is a smooth function and thus defines a Radon measure compactly supported in $\Omega$, and as such it annihilates all solutions of the heat equation on $\mathbf{R}^{n+1}$ but not all solutions on $\Omega$. For, if $H w=0$ on $\mathbf{R}^{n+1}$, then since $v$ has compact support, $\left\langle H^{t} v, w\right\rangle=\langle v, H w\rangle=\langle v, 0\rangle=0$. But if $E$ is a fundamental solution for $H$, then $H E=\delta$, the Dirac meansure at the origin, and so $H E=0$ on $\Omega$. But

$$
\left\langle H^{t} v, E\right\rangle=\langle v, H E\rangle=\langle v, \delta\rangle=v(0,0)=1 \text {. }
$$

Therefore we conclude that the solutions on $\mathrm{R}^{n+1}$ are not dense in the solutions on $\Omega$. Q.E.D. 
4. Remarks. We have dealt with uniform convergence on compact sets in $\Omega$. We could just as well have used the topology of $C^{\infty}(\Omega)$, involving uniform convergence of all derivatives on compact subsets of $\Omega$. These topologies are the same for solutions of the heat equation.

Another result in Malgrange's thesis [4] is that in case the necessary and sufficient condition on $\Omega$ holds, then actually the exponential-polynomial solutions are dense in the solutions on $\Omega$. In fact, one can even use just polynomial solutions since the polynomial $-\xi_{1}^{2}-\cdots-\xi_{n}^{2}-i t$ is irreducible and vanishes at the origin. Also one can use just exponential solutions since this polynomial is irreducible; cf. [2, p. 78].

\section{REFERENCES}

1. G. Hellwig, Partial differential equations: An introduction, Blaisdell, Waltham, Mass., 1964. MR $30 \# 3286$.

2. L. Hörmander, Linear partial differential operators, Die Grundlehren der math. Wissenschaften, Band 116, Academic Press, New York; Springer-Verlag, Berlin, 1963. MR $28 \# 4221$.

3. - An introduction to complex analysis in several variables, Van Nostrand, Princeton, N. J., 1966. MR 34 \#2933.

4. B. Malgrange, Existence et approximation des solutions des équations aux dérivées partielles et des équations de convolution, Ann. Inst. Fourier (Grenoble) 6 (1955/56), 271-355. MR 19, 280. $\# 1420$.

5. W. Rudin, Real and complex analysis, McGraw-Hill, New York, 1966. MR 35

DEPARTMENT OF MATHEMATICS, RICE UNIVERSITY, HOUSTON, TEXAS 77001 